\title{
Satisfacción con el aspecto dental general y tratamien- tos deseados en estudiantes universitarios
}

Clarisse Virginia Díaz Reissner ${ }^{I}$, Milner Iván Morel Barrios*, Nohelia María Pérez Bejarano ${ }^{\mathrm{I}}$

I. Facultad de Odontología. Universidad del Pacífico Privada. Paraguay

Cómo referenciar este artículo/ How to reference this article:
Díaz Reissner CV, Morel Barrios MI, Pérez Bejarano NM. Satisfacción con el aspecto dental general y tratamiento deseados en estudiantes universitarios. Mem. Inst. Investig. Cienc. Salud. $2015 ; 13(2): 18-25$

\section{RESUMEN}

La apariencia dental es considerada un componente de la belleza facial, que al ser restaurada por el odontólogo, podría influir con un efecto positivo en la valoración personal y la calidad de vida. El objetivo del estudio fue determinar la satisfacción general con la apariencia dental en estudiantes de las carreras de Artes Visuales y Diseño de Indumentaria del Instituto Superior de Arte de la Facultad de Arquitectura Diseño y Arte de la Universidad Nacional de Asunción, matriculados en el 2014. Se aplicó un cuestionario que contenía preguntas sobre datos demográficos, satisfacción con el aspecto dental general y con diversos aspectos dentales, así como también sobre el tratamiento estético deseado. La muestra quedó conformada por 108 estudiantes, que corresponde al $49,1 \%$ de la población. Se observó que el $48,2 \%$ se encontraba insatisfecho con el aspecto general de sus dientes, siendo los tratamientos de elección: blanqueamiento $(63,9 \%)$ y ortodoncia $(47,2 \%)$. Los factores predisponentes a la insatisfacción fueron residir en Gran Asunción e interior del país y percibir sus dientes como desalineados. Por otro lado, favoreció la satisfacción el haberse sentirse a gusto con el color de sus dientes.

Palabras Clave: Estética dental, autoimagen, satisfacción del paciente, estudiantes/ arte.

\section{Satisfaction with dental appearance and desired treat- ments in college students}

\section{A B S T R A C T}

Dental appearance is considered one component of facial beauty, which once restored by a dentist might influence generating a positive effect on self-esteem, and quality of life. The aim of this study was to determine general satisfaction of dental appearance in students of Visual Arts and Costume Design of Superior Institute of the Faculty of Architecture, Design and Arts of the National University of Asunción, enrolled in 2014. A questionnaire comprising demographic data, level of satisfaction with general dental appearance, and varied dental aspects, as well as the desired aesthetic dental treatment was used. Sampling was made of 108 students, corresponding to $49.1 \%$ of the population. It was observed that $48.2 \%$ was dissatisfied with the general appearance of their teeth, being the dental treatment options: whitening (63.9\%) and orthodontics $(47.2 \%)$. Predisposing factors to dissatisfaction were the fact of being residing in the "Gran Asuncion" area and the country side, and perceiving one's teeth as misaligned. On the other hand, satisfaction was favored by feeling comfortable with the color of their teeth.

Key words: Dental esthetical, self concept, patient satisfaction, students/art. 


\section{INTRODUCCIÓN}

El aspecto facial y la imagen corporal contribuyen de manera sustancial en la apariencia dental del individuo (1), que al verse desviada de la estética desde la percepción del sujeto, podría terminar afectando la autoestima (2). En este contexto, la apariencia dental se constituye en un componente de la belleza facial, que al ser reestablecido por el odontólogo podría actuar en pos de un efecto positivo en la valoración personal y la calidad de vida (3).

Se debe tener en cuenta que la estética en la Odontología; además del restablecimiento de la sonrisa, se ocupa de corregir las modificaciones en el perfil facial (4), donde los dientes anteriores ocupan un papel preponderante en este contexto, pudiendo verse afectado por diversos factores como el color, tamaño, posición, entre otros (5).

Refiriéndonos a las necesidades estéticas del paciente, se extrae que ellas deben establecerse como prioridad en los tratamientos y compatibilizarla con la función, teniendo en cuenta en ese proceso que puede verse influenciada por factores como sexo, edad, nivel educativo. En referencia al sexo, Akarslan et al. (2009) mencionan que las mujeres tienden a ser más sensibles con respecto a su apariencia y que también la importancia de la apariencia de los dientes disminuye a medida que aumenta la edad y el nivel educativo superior (6).

La búsqueda de las personas por mejorar la apariencia de sus sonrisas ha planteado retos en Odontología estética, en la cual se debe tener en cuenta las expectativas del paciente para conseguir éxito en cualquier proyección de tratamiento.

La población joven podría dar mayor importancia al aspecto físico, por lo que se considera relevante identificar aspectos relativos de satisfacción con la apariencia dental en ellos y en este caso en estudiantes universitarios que además de su condición académica y la corriente de formación en Arte podrían dar mayor importancia a este aspecto. Este hecho podría ayudar entonces a los contenidos de los proyectos de la promoción de la salud bucodental y el tratamiento odontológico, los cuales deberían enfocarse a las necesidades del individuo, y de esa manera contribuir en el bienestar psicosomático; aumentando la sensación de bienestar general y seguridad emocional.

El objetivo del estudio fue determinar la satisfacción general de la apariencia dental y tratamientos deseados en estudiantes de las carreras de Artes Visuales y Diseño de Indumentaria del Instituto Superior de Arte (ISA) de la Facultad de Arquitectura Diseño y Arte de la Universidad Nacional de Asunción (FADA-UNA), matriculados en el 2014.

\section{MATERIALES Y MÉTODO}

El diseño del estudio fue transversal e incluyó una población constituida por estudiantes de las carreras de Artes Visuales y Diseño de Indumentaria del ISA FADAUNA, matriculados en el 2014. Fueron excluidos los estudiantes menores de 18 años de edad o que hayan abandonado la carrera.

El protocolo fue aprobado por el Comité de Investigación de la Universidad del Pacifico Privada.

Se utilizó un cuestionario traducido al español por autorización de su autor (7). El mismo fue sometido al proceso de traducción del inglés al español y viceversa, por tres odontólogos. Se realizó, para su ajuste, una prueba piloto con el fin de evaluar problemas de comprensión y redacción, dando como resultado las adecuaciones en términos de adaptación cultural y terminología más comprensible para la población objeto de estudio.

El cuestionario contenía preguntas sobre datos demográficos, así como también una pregunta sobre la satisfacción del paciente con su aspecto dental general y siete preguntas sobre su satisfacción con diversos aspectos dentales. Además, preguntas 
sobre el tratamiento estético al que desearían someterse (Tabla 1).

Para el cálculo del tamaño de la muestra se utilizó la prevalencia de insatisfacción con la apariencia dental de $52,8 \%$ basado en pacientes atendidos en el Hospital University Sains Malaysia Dental Clinic (7). Para conseguir una precisión del 7\% en la estimación de una proporción, mediante un intervalo de confianza asintótico normal a un $95 \%$ bilateral fue necesario incluir 104 estudiantes universitarios.

Todos los estudiantes fueron invitados a participar de la investigación y la aplicación del cuestionario se realizó en un aula de clase, donde se explicó cada una de las preguntas mediante una presentación en diapositivas que contenían imágenes de referencia, de manera a facilitar la comprensión de las preguntas.

Tabla 1. Cuestionario utilizado en el estudio.

\begin{tabular}{llc}
\hline Sexo: $\square$ Masculino $\square$ Femenino & Edad:_ (años) \\
Procedencia: $\square$ Asunción $\square$ Gran Asunción $\square$ Interior del país & \\
¿Has recibido tratamiento dental en los últimos 6 meses? & $\square \mathrm{Si}$ & $\square \mathrm{No}$ \\
& $\square \mathrm{Si}$ & $\square \mathrm{No}$ \\
& $\square \mathrm{Si}$ & $\square \mathrm{No}$ \\
1. ¿Te sientes satisfecho con el aspecto general de tus dientes? & $\square \mathrm{Si}$ & $\square \mathrm{No}$ \\
2. ¿Te sientes a gusto con el color de tus dientes? & $\square \mathrm{Si}$ & $\square \mathrm{No}$ \\
3. ¿Sientes que tus dientes se aprietan (no pasa el hilo dental)? & $\square \mathrm{Si}$ & $\square \mathrm{No}$ \\
4. ¿Sientes que tus dientes no están bien alineados? & $\square \mathrm{Si}$ & $\square \mathrm{No}$ \\
5. ¿Sientes que tus dientes sobresalen (salen hacia afuera)? & $\square \mathrm{Si}$ & $\square \mathrm{No}$ \\
6. ¿Tiene caries en tus dientes de adelante? & $\square \mathrm{Si}$ & $\square \mathrm{No}$ \\
7. ¿Tienes restauraciones no estéticas en tus dientes de adelante? & $\square \mathrm{Si}$ & $\square \mathrm{No}$ \\
8. ¿Tienes fracturado alguno de tus dientes de adelante? & $\square \mathrm{Si}$ & $\square \mathrm{No}$ \\
9. ¿Qué tratamientos te gustaría realizar para mejorar la apariencia de tus & $\square \mathrm{Si}$ & $\square \mathrm{No}$ \\
dientes? & $\square \mathrm{Si}$ & $\square \mathrm{No}$ \\
a. Frenillo para alinear los dientes (ortodoncia) & $\square \mathrm{Si}$ & $\square \mathrm{No}$ \\
b. Blanqueamiento dental & $\square \mathrm{Si}$ & $\square \mathrm{No}$ \\
c. Coronas (forros o carillas) dentales &
\end{tabular}

En cuanto a gestión y análisis de datos, el cálculo del tamaño de la muestra se realizó con el programa Ene 3.0. Los datos fueron tabulados en Microsoft Excel ${ }^{\circledR}$ 2013, mientras que el análisis estadístico se realizó con el programa R 3.0.3 (8) con los paquetes Baylor Ed Psych (9), Resource Selection (10) y MASS (11).

Para presentar los resultados se utilizó estadística descriptiva mediante frecuencia y porcentaje para las variables cualitativas y media para las cuantitativas, así como también, estadística inferencial utilizando la prueba Chi-cuadrado de Pearson de homogeneidad y el Test Exacto de Fisher para comparar la independencia en cuanto al sexo, edad, procedencia y cada una de las variables que influyen en la insatisfacción, entre los estudiantes satisfechos e insatisfechos con su apariencia dental general, con nivel de confianza del $95 \%$. Las variables que influenciaron la satisfacción con la apariencia dental general fueron determinados mediante análisis de regresión logística múltiple, también utilizando un nivel de confianza del $95 \%$.

\section{RESULTADOS}

De 220 estudiantes matriculados en las carreras de Artes Visuales y Diseño de Indumentaria del ISA de la FADA-UNA en el 2014, la muestra quedó conformada por 108 estudiantes que corresponden al 49,1\%. La edad mínima fue de 18 años y la máxima de 55, siendo la mediana de edad de 23 años.

Aproximadamente la mitad de los estudiantes se encontraba satisfecho con el aspecto 
general de sus dientes, más frecuentemente con la falta de alineamiento de dientes anteriores y el tratamiento mayormente requerido fue el blanqueamiento dental (Tabla 2 ). Cabe destacar que, aunque el $67,6 \%$ declaró haber recibido tratamiento dental en los últimos 6 meses, de igual manera reportaron que se realizarían otros tratamientos odontológicos en el futuro ya que todos manifestaron estar disconformes con por lo menos un aspecto de su apariencia dental general y se realizarían por lo menos un tratamiento dental.

Tabla 2. Características demográficas y bienestar dental de estudiantes del Instituto Superior de Arte de la Facultad de Arquitectura de la Universidad Nacional de Asunción, Paraguay. $2014(n=108)$.

\begin{tabular}{|c|c|c|}
\hline Variables & $\mathbf{n}$ & $\%$ \\
\hline \multicolumn{3}{|l|}{ Demográficas } \\
\hline \multicolumn{3}{|l|}{ Edad (años) } \\
\hline 18 a 23 & 67 & 62,0 \\
\hline 24 a 55 & 41 & 38,0 \\
\hline \multicolumn{3}{|l|}{ Sexo } \\
\hline Femenino & 90 & 83,3 \\
\hline Masculino & 18 & 16,7 \\
\hline \multicolumn{3}{|l|}{ Procedencia } \\
\hline Asunción & 65 & 60,2 \\
\hline Gran Asunción e interior del país & 43 & 39,8 \\
\hline \multicolumn{3}{|l|}{ Dientes } \\
\hline \multicolumn{3}{|c|}{ Satisfecho con la apariencia general } \\
\hline No & 52 & 48,2 \\
\hline $\mathrm{Si}$ & 56 & 51,9 \\
\hline \multicolumn{3}{|l|}{ Satisfecho con el color } \\
\hline No & 54 & 50,0 \\
\hline $\mathrm{Si}$ & 54 & 50,0 \\
\hline \multicolumn{3}{|l|}{ Siente que se aprietan } \\
\hline No & 86 & 79,6 \\
\hline $\mathrm{Si}$ & 22 & 20,4 \\
\hline \multicolumn{3}{|l|}{ Siente que están desalineados } \\
\hline No & 44 & 40,7 \\
\hline $\mathrm{Si}$ & 64 & 59,3 \\
\hline \multicolumn{3}{|l|}{ Siente que sobresalen } \\
\hline No & 78 & 72,2 \\
\hline $\mathrm{Si}$ & 30 & 27,8 \\
\hline \multicolumn{3}{|l|}{ Tiene caries en anteriores } \\
\hline No & 102 & 94,4 \\
\hline $\mathrm{Si}$ & 6 & 5,6 \\
\hline \multicolumn{3}{|c|}{ Tiene restauraciones antiestéticas en anteriores } \\
\hline No & 95 & 88,0 \\
\hline & 13 & 12,0 \\
\hline \multicolumn{3}{|l|}{ Tiene fracturado anterior } \\
\hline No & 97 & 89,8 \\
\hline $\mathrm{Si}$ & 11 & 10,2 \\
\hline
\end{tabular}

Los tratamientos dentales menos deseados por los estudiantes fueron la prótesis dental, ya sea ésta removible o implantes, seguido de corona dental. Aproximadamente la mitad de los estudiantes dijo que se realizaría blanqueamiento dental y tratamiento de ortodoncia (Tabla 3). 
Tabla 3. Tratamiento odontológico deseado por los estudiantes del ISA de la FADA, UNA. Paraguay, $2014(n=108)$

\begin{tabular}{lll}
\hline Variables & $\mathbf{n}$ & $\mathbf{\%}$ \\
\hline Ortodoncia & & \\
No & 57 & 52,8 \\
$\mathrm{Si}$ & 51 & 47,2 \\
Blanqueamiento & & \\
$\mathrm{No}$ & 39 & 36,1 \\
$\mathrm{Si}$ & 69 & 63,9 \\
Corona & & \\
$\mathrm{No}$ & 95 & 88,0 \\
$\mathrm{Si}$ & 13 & 12,0 \\
Restauración estética & & \\
No & 77 & 71,3 \\
Si & 31 & 28,7 \\
Dentadura & & \\
No & 100 & 92,6 \\
$\mathrm{Si}$ & 8 & 7,4 \\
\hline
\end{tabular}

Resultaron variables relacionadas con la satisfacción con la apariencia general de los dientes, la procedencia, ya que los asuncenos se encontraron mayormente satisfechos que los residentes en Gran Asunción e interior del país con el color de sus dientes así como también con la presencia de dientes anteriores desalineados, encontrándose en mayor proporción insatisfechos los segundos (Tabla 4).

Se aplicó un análisis de regresión logística con las variables estadísticamente significativas en el análisis bivariado, obteniéndose como resultado un modelo de estimación con buen ajuste: prueba de Hosmer y Lemeshow $\left(X^{2}=6,317 ; p=0,612\right) ; R^{2}$ de Negelkerke fue de 0,40 y tasa de clasificación correcta del 77,1\%.

Los estudiantes procedentes de Gran Asunción e interior del país con respecto a aquellos que son de Asunción tuvieron 4 veces más probabilidad de estar insatisfechos con el aspecto general de sus dientes. Así también, los estudiantes que consideraban que sus dientes se encontraban desalineados con respecto a aquellos que se encontraban conformes con la alineación de sus dientes tuvieron la misma probabilidad de insatisfacción. Encontrarse a gusto con el color de los dientes actuó como un factor que predispuso a que el estudiante se encuentre satisfecho con el aspecto general de sus dientes, exactamente 5 veces más con respecto a aquellos que no se sentían conformes con el color de los mismos (Tabla 5). 
Tabla 4. Relación entre la satisfacción con la apariencia general de los dientes y variables demográficas y bienestar dental de los estudiantes del ISA de la FADA, UNA. Paraguay, 2014

\begin{tabular}{|c|c|c|c|}
\hline Variables & $\begin{array}{l}\text { Satisfecho } \\
n=56\end{array}$ & $\begin{array}{l}\text { Insatisfecho } \\
n=52\end{array}$ & valor p \\
\hline \multicolumn{4}{|l|}{ Demográficas } \\
\hline \multicolumn{4}{|l|}{ Edad } \\
\hline 18 a 23 años & $37(66,0)$ & $30(57,7)$ & \multirow{2}{*}{0,370} \\
\hline 24 a 55 años & $19(33,9)$ & $22(42,3)$ & \\
\hline \multicolumn{4}{|l|}{ Sexo } \\
\hline Femenino & $47(83,9)$ & $43(82,7)$ & \multirow{2}{*}{0,863} \\
\hline $\begin{array}{l}\text { Masculino } \\
\text { Procedencia }\end{array}$ & $9(16,1)$ & $9(17,3)$ & \\
\hline $\begin{array}{l}\text { Procedencia } \\
\text { Asunción }\end{array}$ & $43(76,8)$ & $22(42,3)$ & \multirow[b]{2}{*}{$<0,001^{*}$} \\
\hline Gran Asunción e interior del país & $13(23,2)$ & $30(57,7)$ & \\
\hline \multicolumn{4}{|l|}{ Dientes } \\
\hline Satisfecho con el color & & & \multirow{3}{*}{$<0,001^{*}$} \\
\hline No & $18(32,1)$ & $16(30,8)$ & \\
\hline $\mathrm{Si}$ & $38(67,9)$ & $36(69,2)$ & \\
\hline \multicolumn{4}{|l|}{ Siente que se aprietan } \\
\hline No & $48(85,7)$ & $38(73,1)$ & \multirow{2}{*}{0,103} \\
\hline $\mathrm{Si}$ & $8(14,3)$ & $14(26,9)$ & \\
\hline \multicolumn{4}{|l|}{ Siente que están desalineados } \\
\hline No & $33(59,0)$ & $11(21,2)$ & \multirow{2}{*}{$<0,001^{*}$} \\
\hline $\mathrm{Si}$ & $23(41,1)$ & $41(78,8)$ & \\
\hline \multicolumn{4}{|l|}{ Siente que sobresalen } \\
\hline No & $45(80,4)$ & $33(63,5)$ & \multirow{2}{*}{0,050} \\
\hline $\mathrm{Si}$ & $11(19,6)$ & $19(36,5)$ & \\
\hline \multicolumn{4}{|l|}{ Tiene caries en anteriores } \\
\hline No & $54(96,4)$ & $48(92,3)$ & \multirow{2}{*}{$0,425+$} \\
\hline $\mathrm{Si}$ & $2(3,6)$ & $4(7,7)$ & \\
\hline \multicolumn{4}{|c|}{$\begin{array}{l}\text { Tiene restauraciones no estéticas en anterio- } \\
\text { res }\end{array}$} \\
\hline No & $51(91,1)$ & $44(84,6)$ & \multirow{2}{*}{0,303} \\
\hline $\mathrm{Si}$ & $5(8,9)$ & $8(15,4)$ & \\
\hline \multicolumn{4}{|l|}{ Tiene fracturado anterior } \\
\hline $\begin{array}{l}\mathrm{No} \\
\mathrm{Si}\end{array}$ & $\begin{array}{l}51(91,1) \\
5(8,9)\end{array}$ & $\begin{array}{l}46(88,5) \\
6(11,5)\end{array}$ & 0,654 \\
\hline
\end{tabular}

*Estadísticamente significativo al 0,05 +Test Exacto de Fisher

Tabla 5. Regresión logística entre satisfacción con el aspecto general de los dientes con variables demográficas y bienestar dental de los estudiantes del ISA de la FADA, UNA. Paraguay, 2014

\begin{tabular}{llllllll}
\hline Variables & $\boldsymbol{\beta}$ & $\begin{array}{l}\text { Error } \\
\text { estándar }\end{array}$ & $\mathbf{z}$ & $\begin{array}{l}\text { Valor } \\
\mathbf{p}\end{array}$ & $\mathbf{E x p ( \boldsymbol { \beta } )}$ & IC95\% para $\exp (\boldsymbol{\beta})$ \\
\hline Intercepto & 0,769 & 0,456 & 1,688 & 0,091 & -- & -- & -- \\
$\begin{array}{l}\text { Procedencia [Gran } \\
\text { Asunción e interior] }\end{array}$ & $-1,434$ & 0,486 & $-2,948$ & 0,003 & 0,238 & 0,088 & 0,604 \\
$\begin{array}{l}\text { Desalineación [Si] } \\
\text { A gusto con color }\end{array}$ & $-1,512$ & 0,485 & $-3,116$ & 0,002 & 0,220 & 0,082 & 0,557 \\
[Si] & 1,605 & 0,475 & 3,377 & 0,001 & 4,979 & 2,016 & 13,1736 \\
\hline
\end{tabular}

*Estadísticamente significativo al 0,05. 


\section{DISCUSIÓN}

La evaluación de parámetros relacionados con la personalidad humana actualmente son elementos esenciales, que influyen en la estética humana; por lo que no se pueden subestimar. El odontólogo que incluya esa percepción en el tratamiento propuesto para el paciente, permitirá cumplir con las exigencias individuales contribuyendo a la autosatisfacción de la estética.

El porcentaje de insatisfacción general de adultos jóvenes de las carreras de Artes Visuales y Diseño de Indumentaria del Instituto Superior de Arte (ISA) de la Facultad de Arquitectura Diseño y Arte de la Universidad Nacional de Asunción (FADA-UNA) resultó similar al estudio realizado por Akarslan et al. (6) en el 2009 en pacientes ambulatorios turcos con un rango de edad de 16 a 70 años (media 31,8 de 12,7) atendidos en una escuela de Odontología, en donde obtuvieron un 48,5\% de insatisfacción general, luego de aplicárseles un cuestionario de autopercepción; previo a los tratamientos a realizarse en dientes anteriores. Por su parte comparado con el $52,8 \%$ en el estudio de corte trasversal realizado por Tin-Oo et al. (2011) (7) en 235 pacientes adultos de una edad media de 31,5 años (DE 13,0) malasios que acudieron al Hospital University Sains Malaysia Dental Clinic, aproximadamente la mitad de los estudiantes universitarios reportaron estar insatisfechos con el aspecto general de sus dientes. Nuestros valores fueron ligeramente inferiores a lo demostrado por los pacientes adultos malasios.

La insatisfacción con la apariencia fue referido por Azodo et al. en el 2014 (1) en $20,6 \%$ en estudiantes universitarios de pregrado de Industrias Financieras nigerianos de la Universidad de Benín; de la misma manera se reportó una insatisfacción dental en solo un $12,1 \%$ en 3.384 adultos británicos mayores de 55 años en el estudio de Alkhatib et al. (12) que utilizó un cuestionario cara a cara con los sujetos como parte de una encuesta nacional sobre percepción de descoloración de dientes, el cual contenía imágenes de dientes con diferentes colores a los cuales los sujetos deberían asignarle una valoración además de responder preguntas sobre la autopercepción.

Nuestros hallazgos también superan al 4,9\% obtenido por Afroz et al. (3) en el 2013 a través de un cuestionario autoadministrado en estudiantes musulmanes de diferentes corrientes educativas, a excepción de los estudiantes de Odontología de la Universidad de Aligarh. De hecho, diversos estudios han reportado variación con la edad, encontrándose mayor insatisfacción en los jóvenes que los adultos $(5,12)$.

Los factores dentales que podrían influir en la insatisfacción fueron la preocupación por el color de los dientes y la falta de alineación de los mismos, resultado coincidente al reportado por Akarslan et al., pacientes turcos de diversas edades (6) que fueron atendidos en la escuela de Odontología y reportaron estar insatisfechos con el color de sus dientes en un $55,1 \%$ y por el ya mencionado estudio de Tin-Oo et al., (7), encontrándose insatisfacción con el color de los dientes en proporción 56,2\%. Cabe destacar, que conforme el estudio realizado por Borges et al. (13) todos los tipos de maloclusión estuvieron asociados con la insatisfacción con la apariencia dental y gingival, en adolescentes brasileños de 15 a 19 años $(n=16.126)$ comparado con el $40 \%$ hallado en nuestra población estudiada.

En cuanto a los factores socio-demográficos que pudieran influir en la satisfacción de la apariencia dental, en este estudio el sexo no resultó estadísticamente significativo, sin embargo, las mujeres en el estudio de Tin-Oo et al. (7), fueron significativamente más críticas en la apreciación de su apariencia dental ( $\mathrm{OR}=2,70 ; 95 \% \mathrm{CI}: 1,51-4,82$ ).

Con respecto al tratamiento deseado, los estudiantes paraguayos consideraron en primer lugar el blanqueamiento dental, coincidente con el estudio de Tin-Oo et al.(7). Es de esperar que por ser en su mayoría adultos jóvenes, la necesidad de realizarse prótesis dental o coronas dentales debería ser más baja. Posiblemente por la tendencia a asociar la necesidad de dichos tratamientos con un estado deteriorado de salud bucal que se presenta en adultos mayores. 
Se encontró en este trabajo que la procedencia, el color y la desalineación se asociaron con la autopercepción del aspecto general y a todo lo expuesto se podría agregar que la sociedad espera recibir en un futuro una atención dental de odontólogos de alto nivel profesional, basados en los principios de ética incluyendo las necesidades de cada individuo y no con enfoque técnico solamente, volviendo con esto la atención en integral con enfoque biopsicosocial (14).

Se recomienda continuar el estudio, con individuos de otras áreas, de manera a comparar la existencia de asociación entre las consideraciones hacia la estética de los individuos incluidos en este estudio.

\section{REFERENCIAS BIBLIOGRAFICAS}

1. Azodo C, Ogbomo A. Self-evaluated dental appearance satisfaction among young adults. Ann Med Heal Sci Res. 2014;4 (4):603-7.

2. Peres $\mathrm{SH}$, Goya S, Cortellazzi KL, Ambrosano GM, Meneghim M de C, Pereira AC. Self-perception and malocclusion and their relation to oral appearance and function. Ciên Saúde Colet. 2011 Oct;16(10):405966.

3. Afroz S, Rathi S, Rajput G, Rahman SA. Dental esthetics and its impact on psychosocial well-being and dental self confidence: A campus based survey of North Indian University students. J Indian Prosthodont Soc. $2013 ; 13(4): 455-60$.

4. Manipal S, Mohan CSA, Kumar DL, Cholan PK, Ahmed A, Adusumilli P. The importance of dental aesthetics among dental students assessment of knowledge. J Int Soc Prev Community Dent. 2014;4(1):48-51.

5. Lajnert V, Pavičić DK, Gržić R, Kovač Z, Pahor $D$, Kuis $D$, et al. Influences of age and maxillary anterior teeth status on patient's satisfaction with dental appearance and tooth colour. Gerodontology. 2012 Jun;29 (2):e674-9.

6. Akarslan ZZ, Sadik B, Erten H, Karabulut E. Dental esthetic satisfaction, received and desired dental treatments for improvement of esthetics. Indian J Dent Res. 2009; 20 (2):195-200.

7. Tin-Oo M, Saddki N, Hassan N. Factors influencing patient satisfaction with dental appearance and treatments they desire to improve aesthetics. Bmc Oral Heal. 2011;11 (1):1-8.
8. R Development Core Team. R: A language and environment for statistical computing [Internet]. Vienna: R Foundation for Statistical Computing; 2011. /citado 10 feb 2015/ Available from: http://www.R-project.org/

9. Beaujean AA. R Package for Baylor University educational psychology quantitative courses [Internet]. CRAN; 2012. /citado 10 feb 2015/. Available from: http://CRAN.Rproject.org/package $=$ BaylorEdPsych

10.Lele SR, Keim JL, Solymos P. Resource selection: Resource selection (Probability) functions for use-availability data [Internet]. 2014. /citado 12 may 2015/. Available from: h t t p : / / CRA N.R - project.org / package $=$ ResourceSelection

11.Venables WN, Ripley BD. Modern Applied Statistics with $S$ [Internet]. New York: Springer; 2002. Available from: http:// www.stats.ox.ac.uk/pub/MASS4

12. Alkhatib $M N$, Holt R, Bedi R. Age and perception of dental appearance and tooth colour. Gerodontology. 2005 Mar;22(1):32-6.

13.Borges CM, Peres MA, Peres KG. Association between malocclusion and dissatisfaction with dental and gingival appearance: study with brazilian adolescents. Rev Bras Epidemiol. 2010 Dec;13(4):713-23.

14.Ozar DT. Professionalism: challenges for dentistry in the future. J Forensic Odontostomatol. 2012 Nov;30 Suppl 1:72-84. 